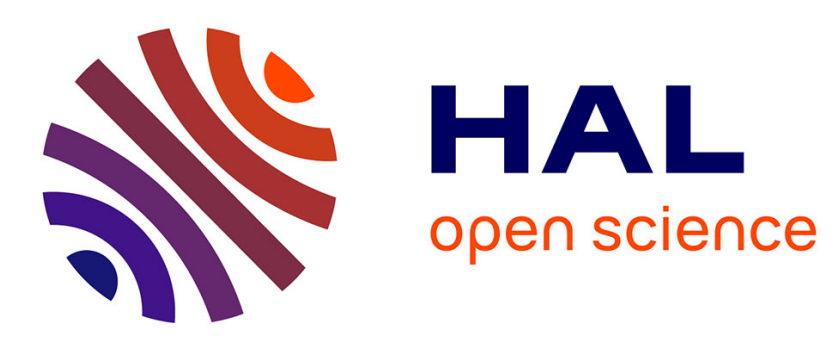

\title{
Performance Model for 4G/5G Networks Taking into Account Intra- and Inter-Cell Mobility of Users
}

\author{
Bruno Baynat, Narcisse Nya Kamtchoum
}

\section{To cite this version:}

Bruno Baynat, Narcisse Nya Kamtchoum. Performance Model for 4G/5G Networks Taking into Account Intra- and Inter-Cell Mobility of Users. 41st IEEE International Conference on Local Computer Networks (LCN 2016), IEEE, Nov 2016, Dubaï, United Arab Emirates. pp.212-215, 10.1109/LCN.2016.45 . hal-01620458

\section{HAL Id: hal-01620458 https://hal.science/hal-01620458}

Submitted on 20 Oct 2017

HAL is a multi-disciplinary open access archive for the deposit and dissemination of scientific research documents, whether they are published or not. The documents may come from teaching and research institutions in France or abroad, or from public or private research centers.
L'archive ouverte pluridisciplinaire HAL, est destinée au dépôt et à la diffusion de documents scientifiques de niveau recherche, publiés ou non, émanant des établissements d'enseignement et de recherche français ou étrangers, des laboratoires publics ou privés. 


\section{Performance Model for 4G/5G Networks Taking Into Account Intra- and Inter-Cell Mobility of Users}

\author{
Bruno Baynat \\ Sorbonne Universits, UPMC Univ Paris 06, \\ CNRS, LIP6 UMR 7606, \\ 4 place Jussieu 75005 Paris \\ E-mail: bruno.baynat@lip6.fr
}

\author{
Nya Narcisse \\ Sorbonne Universits, UPMC Univ Paris 06, \\ CNRS, LIP6 UMR 7606, \\ 4 place Jussieu 75005 Paris \\ E-mail: narcisse.nya@lip6.fr
}

\begin{abstract}
In this paper, we investigate the influence of intraand inter-cell mobility of users on performance of $4 \mathrm{G} / \mathbf{5 G}$ cellular networks, such as LTE and LTE-A. To this end, we develop a multi-class PS queue model that captures mobility of users between zones of a cell and between cells, through a simple mobility model, that is decoupled from the cell model itself, enabling to directly apply the approach to more realistic mobility patterns. We first show that this model is consistent with known analytical bounds corresponding to a system with either static users or users having an infinite speed. We then compare our model to simulations for more realistic speeds, and show that it provides user and cell performance with a very good accuracy. The outcomes of our model confirm that mobility may improve both users and cells performance, and enable to quantify the gain as a function of users speed.
\end{abstract}

\section{INTRODUCTION}

In LTE, like in other wireless communication systems, Adaptive Modulation and Coding (AMC) has become a standard approach. The idea behind AMC is to dynamically adapt the modulation and coding scheme to the channel conditions so as to achieve the highest spectral efficiency at all times [1]. As such, modulation and/or coding can change with time for a given user depending on its location and its channel quality. This is especially true when users are mobile, highlighting the influence of mobility on user and cell performance. The resulting variations in the transmission rates is exploited by opportunistic schedulers to increase overall throughput of data transmissions [2], [3], [4]. But even schedulers with fair resource sharing strategy can take advantage of users mobility.

The fact that mobility may improve performance has already been observed in the literature (e.g., in [5], [6], [7], [8], [9]). These papers mainly present theoretical properties and performance bounds. For example, in [5], authors identify two limit regimes of infinitely fast and infinitely low channel variations, and show that these limit regimes provide simple

* This work has been carried out in the framework of IDEFIX project, funded by the ANR under the contract number ANR-13-INFR-0006. bounds on performance at a flow-level. [6] also develops lower and upper bounds for the flow-level performance measures, and show that mobility tends to increase the overall capacity of the network. In [7], authors analyze networks with several interacting base stations, and show that mobility increases the stability region of the system. Authors of [10] model an OFDMA system jointly using Proportional Fairness (PF) and Hierarchical Modulation (HM), by a multi-dimensional Markov chain, and show that in the presence of HM, a simple cyclic service, such as Round Robin, yields better performance than PF. The most related work is [11], in which authors assess the impact of users mobility on cell performance, under a fair and an opportunistic scheduling scheme. They show in particular that under both scheduling policies, mobility improves throughput performance at cell edge. But as the Markovian process associated with their model is no longer reversible in the case where mobile users fairly share resources, they can only develop closed-form expressions in two limiting cases, namely when users are static and when users have a theoritecal infinite speed. For more realistic speeds, their analysis relies on the numerical solution of multi-dimensional Markov chains.

In this paper we develop a multi-class Processor Sharing (PS) queue model, that captures mobility of users through the distribution of the time a given user physically stays in the different coding zones of cells. Contrarily to previous studies, our model does not rely on the numerical analysis of complex Markov chains, or on limiting assumptions such as infinite speed of users, and as such is one of the first tractable and accurate approximations for 4G/5G cellular networks with mobile users. The originality of the approach is to decouple the mobility model from the cell model itself, by relating input parameters of the PS queue to physical mobility parameters. We show that our model is consistent with aforementioned analytical bounds for realistic speeds of users. Thanks to our model, we quantify the gain of speed on both the performance of the cell and the end-to-end performance of users, and investigate the influence of intra- and inter-cell mobility. 
The paper is organized as follows. Section II presents system and trafic assumptions used in the model. Section III develops the PS queue model and all performance parameters of interest. The model is validated through simulation in Section IV that also investigates the impact of users speed on performance. Finally, Section V concludes the paper.

\section{SYSTEM AND TRAFFIC ASSUMPTIONS}

\section{A. The system: a LTE macrocell divided into J zones}

We consider a LTE macrocell with a round robin scheduling discipline. For a given number of active users, resources (Resource Blocks in LTE) are equally divided among users. A user that is alone in the cell will have different bit rates if he is close to the base station, compared to the case where he is far from it. The cell can thus be divided into $J$ zones of equal radio conditions, or classes, each characterized by an achievable throughput, i.e., a throughput that can be achieved by a user when scheduled by the base station [12]. A user of class $j, j=1, \ldots, J$, i.e., currently present in zone $j$, will obtain a throughput $C_{j}$, if he is alone in the whole cell. Even if it is not necessary for the developments in our work, we can assume as, e.g., in [13], that the $J$ zones form concentric circles of radius $R_{j}$, where zone 1 is the central zone and zone $J$ is the peripheral zone.

\section{B. Traffic assumption and mobility model}

We consider that connection demands arrive to the cell according to a Poisson process of rate $\Lambda$. We assume that a user that carries a new connection demand has a probability $p_{j}$ to be initially of class $j$ and thus to start its download in zone $j$. As a result, new connection demands arrive in zone $j$ according to a Poisson process with a rate $\Lambda_{j}=p_{j} \Lambda$.

Contrarily to previous classical works on PS queue models (e.g., [12]), we assume that users are mobile, and can thus change zone during their download or leave the cell before completing their transfer. The mobility model we consider is the following. It is important to emphasize that it is a physical mobility model of users that is decoupled from the utilization of the resources of the cell by users. We denote by $\Theta_{j}$ the physical sojourn time of a user in zone $j$ at each visit of the zone, that is, the time duration he physically stays in zone $j$ starting form the moment he appears in zone $j$ (either because he begins its download in zone $j$, or because he enters zone $j$ from another neighboring zone or cell), and ending when he leaves zone $j$ (still being active or not) for another neighboring zone or leave definitively the cell. We then define $P_{j k}$, the probability that a user that physically (i.e., active or not) exists zone $j$, move to a neighboring zone $k$. If the outside of the cell is labelled $0, P_{j 0}$ is the probability that a user exits the cell from zone $j$, and $P_{0 j}$ is the probability that a user enters the cell by zone $j$. Note that when zones form concentric circles, users can only leave and enter the cell from zone $J$.

We assume that each new connection demand (regardless of its initial class) brings an identically distributed volume $\Sigma$ of data to be downloaded. As soon as a new request arrives, it triggers the start of a new data transmission (in the zone where the request appears). This transmission ends either because the user has completed its transfer before leaving the cell (whatever the number of zones he has visited meanwhile), or because he has left the cell before completing its transfer. As a result, the volume actually transferred by a given user in the cell is, in average, less than $\Sigma$.

We consider in this paper that random variables $\Sigma$ and $\Theta_{j}$ are exponentially distributed. The first assumption is necessary for the derivations presented below. As a matter of fact, and because of the memoryless property of the exponential distribution, assuming an exponential volume enables us to forget the amount of data already transferred by a user in previous zones, as long as he is still active and moves to another zone or to another cell. This drastically simplify the analysis. On the other hand, assuming exponential sojourn time in each zone is only made for simplification purposes, and other more realistic distributions can be considered.

\section{Estimation of parameters when zones are concentric circles}

We now consider the special case where the $J$ zones form concentric circles of radius $R_{j}$, and see how we can estimate the traffic and mobility parameters. First, the surface of zone $j$ is $S_{j}=\pi\left(R_{j}{ }^{2}-R_{j-1}{ }^{2}\right)$, for $j>1$, and $S_{1}=\pi R_{1}{ }^{2}$. If arrivals of new connection demands are uniformly distributed over the whole surface of the cell, the probability $p_{j}$ that a new connection demand appears in zone $j$, is proportional to the surface of zone $j$ :

$$
p_{j}=\frac{S_{j}}{\pi R_{J}^{2}} .
$$

Concerning the mean sojourn time in zone $j, \mathbb{E}\left(\Theta_{j}\right)$, we can reasonably assume that it is proportional to the square root of the surface of the zone and inversely proportional to the speed $V$ of users:

$$
\mathbb{E}\left(\Theta_{j}\right)=K \frac{\sqrt{S_{j}}}{V},
$$

When zones form concentric circles, a user that physically exists zone $j, j=2, \ldots, J-1$, has a probability $P_{j-1}$ to move to zone $j-1$, and a probability $P_{j j+1}$ to move to zone $j+1$ (with, of course, $P_{j-1}+P_{j+1}=1$ ). For zone 1, obviously, $P_{12}=1$. And from zone $J$, a user can either move back to zone $J-1$ with a probability $P_{J} J_{-1}$, or exit the cell with a probability $P_{J 0}$. All these probabilities clearly depend both on the radius $R_{j}$ of zones and on the real mobility of users. However, without additional assumptions on physical 
mobility of users, we can use the following approximation:

$$
P_{j-1}=\frac{R_{j-1}}{2 R_{j}}, j>1 .
$$

This is a linear approximation that respects obvious limits: when $R_{j-1} \rightarrow 0, P_{j-1} \rightarrow 0$, and when $R_{j-1} \rightarrow R_{j}$, $P_{j j-1} \rightarrow \frac{1}{2}$.

Note that probabilities $p_{j}$ and $P_{i j}$, as well as mean sojourn times $\mathbb{E}\left(\Theta_{j}\right)$, are input parameters for our PS queue model. Any alternative expressions resulting from a realistic physical mobility model of users, can be alternately used without changing the development presented below.

\section{MODEL}

\section{A. Model of an isolated cell}

To simplify the presentation, we first consider in this section the cell as isolated from the outside, and do not explicitly take into account active users that make a handover from neighboring cells. This assumption will be relaxed in Section III-B.

The model represents the cell occupancy by a multi-class Processor Sharing queue, as illustrated in Figure 1. Each class corresponds to a zone of the cell. Customers of class $j$ arrive to the queue according to Poisson process of rate $\lambda_{j}, j=1, \ldots, J$. It is important to note that, contrarily to [14], $\lambda_{j}$ is different from the rate $\Lambda_{j}$ of new connection requests that appear in zone $j$, as it must include arrival of users that move from the other zone while still being active. If we denote by $\Lambda_{i j}$ the average number of active users moving from zone $i$ to zone $j$ by unit of time, we have:

$$
\left\{\begin{array}{l}
\lambda_{1}=\Lambda_{1}+\Lambda_{21} \\
\lambda_{j}=\Lambda_{j}+\Lambda_{j-1 j}+\Lambda_{j+1 j}, j=2, \ldots, J-1 \\
\lambda_{J}=\Lambda_{J}+\Lambda_{J-1 J}
\end{array}\right.
$$

Note that, relaxing the assumption that the cell is isolated from the outside, will consist in adding to $\lambda_{J}$ a term that will represent the rate of active users making a handover from the outside. For the time being, we can just consider that this rate is part of the supposedly known rate $\Lambda_{J}$ of new connection demands appearing in zone $J$.

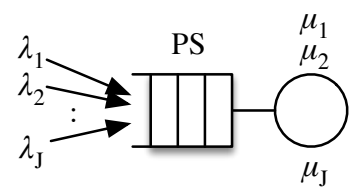

Fig. 1: PS queue model

Class- $j$ rate can in turn be expressed as in [14]:

$$
\mu_{j}=\frac{C_{j}}{\bar{x}_{j}},
$$

where $\bar{x}_{j}$ is defined as the average number of bits transferred by an active user in zone $j$, for each visit of the zone, i.e., between the moment he enters the zone (or appears in the zone) and the moment he leaves it (or finishes his transfer in the zone)

Before going further, it is important to emphasize that this PS queue is always stable, i.e., there is no stability condition for this system. This can be intuitively explained by the fact that all users are mobile and will eventually leave the cell after a finite time. When the rate of news connection demands increases and tends to infinity, users are very likely to leave the cell before downloading a single bit.

As a result, we are left to estimate all the input parameters of the PS queue, namely $\lambda_{j}$ and $\mu_{j}$, for $j=1, \ldots, J$, or more precisely all $\Lambda_{i j}$ and $\bar{x}_{j}$. Note that in [14], only one parameter was left to estimate.

Given parameters $\lambda_{j}$ and $\mu_{j}$, standard results for the stationary multi-class Processor Sharing queues can be readily applied to calculate the average throughputs $\bar{\gamma}_{j}$ obtained by users in zone $j$ during their transfer, that is,

$$
\bar{\gamma}_{j}=C_{j}(1-\rho),
$$

where $\rho=\sum_{j=1}^{J} \rho_{j}$ and $\rho_{j}=\frac{\lambda_{j}}{\mu_{j}}$.

In order to estimate the first missing parameters $\bar{x}_{j}$, required in the expression of $\mu_{j}$, we use the methodology developed in [14] for a cell with a single zone, and apply it to each zone individually. As shown in [14] in the special case where $\Sigma$ and $\Theta_{j}$ are exponentially distributed, $\bar{x}_{j}$ is related to the average throughput $\bar{\gamma}_{j}$ obtained by users in zone $j$ and to the average time $\mathbb{E}\left(\Theta_{j}\right)$ a user physically spends in zone $j$, as:

$$
\bar{x}_{j}=\frac{\mathbb{E}(\Sigma) \mathbb{E}\left(\Theta_{j}\right) \bar{\gamma}_{j}}{\mathbb{E}(\Sigma)+\mathbb{E}\left(\Theta_{j}\right) \bar{\gamma}_{j}} .
$$

We now need to estimate the missing parameters $\Lambda_{i j}$ appearing in the expressions of $\lambda_{j}$. If we denote by $h_{j}$ the handover probability from zone $j$, i.e., the probability that an active user in zone $j$ leaves the zone without having finished its transfer, we can express $\Lambda_{j j+1}$ and $\Lambda_{j j-1}$ as:

$$
\left\{\begin{array}{l}
\Lambda_{j j+1}=\lambda_{j} h_{j} P_{j j+1}, j=1, \ldots, J-1 \\
\Lambda_{j j-1}=\lambda_{j} h_{j} P_{j j-1}, j=2, \ldots, J
\end{array}\right.
$$

The handover probabilities $h_{j}$ can in turn be expressed as:

$$
h_{j}=\int_{0}^{+\infty} \mathbb{P}\left[\Theta_{j} \leq \frac{y}{\bar{\gamma}_{j}}\right] f_{\Sigma}(y) \mathrm{d} y .
$$

Assuming that $\Sigma$ and $\Theta_{j}$ are exponentially distributed, the previous integral readily gives [14]:

$$
h_{j}=\frac{\mathbb{E}(\Sigma)}{\mathbb{E}(\Sigma)+\mathbb{E}\left(\Theta_{j}\right) \bar{\gamma}_{j}} .
$$

We finally end up with a system of 6 dependent equations $(4-8,10)$ that will be solved using a fixed-point iterative technique. 


\section{B. Extension to take into account handovers from the outside of the cell}

Consider the cell as isolated is not realistic. Indeed, if some users can exit the cell without having completed their transfer (which is what we consider from the beggining), it is normal to consider that some users can enter the cell from the outside, with some data volume remaining to be transferred. In order to account for outside handovers, we can simply add to the rate $\lambda_{J}$ of the external zone of the cell, the rate of users making a handover from the outside, denoted as $\Lambda_{0 J}$ :

$$
\lambda_{J}=\Lambda_{J}+\Lambda_{J-1 J}+\Lambda_{0 J}
$$

Now, the question becomes: how can we estimate $\Lambda_{0 J}$ ? The solution mentioned earlier, that consists in considering that $\Lambda_{0 J}$ is a known input parameter is not satisfying. We now propose an alternative solution that consists in assuming that the considered cell is involved in a network of statistically equivalent cells. If this is true, the average number of active users by unit of time that make a handover from the cell to the outside, must be equal to the average number of active users by unit of time that make a handover from the outside to the cell. In other words, we must have $\Lambda_{0 J}=\Lambda_{J 0}$, and this last quantity can simply be related to the handover probability of zone $J$ of the considered cell as: $\Lambda_{J 0}=\lambda_{J} h_{J} P_{J 0}$. As a result, to take into account a cell involved in a network of equivalent cells, the last equation of system 4 must be replaced by:

$$
\lambda_{J}=\frac{1}{1-h_{J} P_{J 0}}\left(\Lambda_{J}+\Lambda_{J-1 J}\right)
$$

However, it is very important to note that this system taking into account handovers from the outside, is no longer always stable. As shown in [11], the stability condition of this system is independent of the speed $V$ of users and is equivalent to the stability condition of a system where users have an infinite speed. It can be expressed as: $\Lambda<\frac{C_{\infty}}{\mathbb{E}(\Sigma)}$, where $C_{\infty}$ is the equivalent capacity of the system where users have an infinite speed and is defined in the Section III-D.

\section{Performance of users in the cell}

We now see how we can derive from the model the performance of an active user in the considered cell. First, we can redraw the PS queue model as in Figure 2 for the special case where $J=2$, by clearly showing the feedback loops of active users making a Handover from one zone to the other one, and active users coming from the outside.

Let us denote by $\lambda$ the total arrival rate of connection demands in a cell. $\lambda$ is different from the rate $\Lambda$ of new connection requests that appear in cell, as it must include users that move from the outside of the cell, while still being active:

$$
\lambda=\Lambda+\Lambda_{0 J}=\Lambda+\Lambda_{J 0} .
$$

From classical results of PS queues, we can calculate the average number of customers of each class, corresponding to the average number of active users in each zone:

$$
\bar{Q}_{j}=\frac{\rho_{j}}{1-\rho},
$$

and derive from Little law, the average time $\bar{R}$ spent by an active user in the cell, starting from the moment where a new active user appears in the cell (either because of a new connection demand or by handover of an active user from the outside), and ending either when the user finishes its transfer in the cell before leaving it, or leave the cell before the completion of its transfer:

$$
\bar{R}=\frac{\sum_{j=1}^{J} \bar{Q}_{j}}{\lambda} .
$$

We define $q_{j}$ as the probability that an active user is in zone $j$ :

$$
q_{j}=\frac{\bar{Q}_{j}}{\sum_{i=1}^{J} \bar{Q}_{i}} .
$$

We can then calculate the average throughput $\bar{\gamma}$ obtained by an active user during it whole sojourn in the cell:

$$
\bar{\gamma}=\sum_{j=1}^{J} q_{j} \bar{\gamma}_{j} .
$$

From $\bar{\gamma}$ and $\bar{R}$, we can derive $\bar{X}$, the total number of bits transferred, in average, by a user during its whole sojourn in the cell:

$$
\bar{X}=\bar{\gamma} \bar{R}
$$

Of course, $\bar{X} \leq \mathbb{E}(\Sigma)$.

We finally estimate the global handover probability $H$, i.e., the probability that an active user leaves the cell before completing its transfers, whatever the number of zones he has visited meanwhile. $H$ is calculated as the ratio between the average number of active users leaving the cell by unit of time (from zone $J$ ) and the total number of new active users that appear in the cell by unit of time:

$$
H=\frac{\bar{Q}_{J} \frac{P_{J 0}}{\mathbb{E}\left(\Theta_{J}\right)}}{\lambda} .
$$

\section{Performance bounds}

The performance of the system we consider with mobile users physically moving with some speed $V$, is bounded by that of two systems. In the first one, users are static $(V=0)$, and in the second one, users have an infinite speed $(V=\infty)$.

The model corresponding to the first system where users are static, is the classical multi-class PS queue with parameters $\lambda_{j}=\Lambda_{j}$ and $\mu_{j}=\frac{C_{j}}{\mathbb{E}(\Sigma)}$. It is known to be equivalent to a single-class PS queue with an arrival rate $\lambda=\sum_{j=1}^{J} \lambda_{j}$, and a service rate $\mu$ equal to the harmonic mean of each service rate: $\frac{1}{\mu_{S}}=\sum_{j=1}^{J} \frac{p_{j}}{\mu_{j}}, p_{j}$ being the probability that a new 


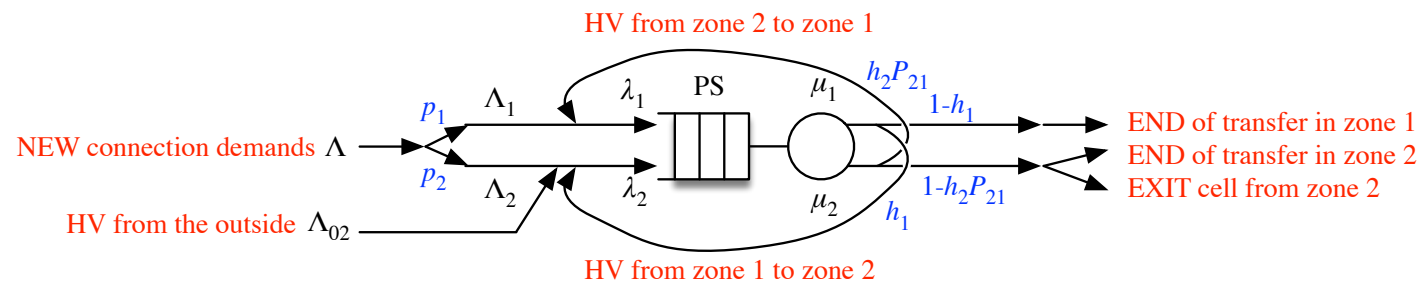

Fig. 2: Illustration of handovers between zones and from the outside

connection demand appears in zone $j$. It corresponds to a cell with a single zone having an equivalent capacity $C_{S}$ equal to the harmonic mean of $C_{j}$ :

$$
\frac{1}{C_{S}}=\sum_{j=1}^{J} \frac{p_{j}}{C_{j}} .
$$

In this system, static users obtain an average throughput $\bar{\gamma}_{S}$ during their transfer, given by:

$$
\bar{\gamma}_{S}=C_{S}(1-\rho),
$$

where $\rho=\frac{\lambda}{\mu_{S}}$, as long as the stability condition $\lambda<\mu_{S}$ is satisfied. Figure 3 illustrates this first bound in red.

As shown in [11], the second system where users have an infinite speed, is equivalent to a single-class PS queue with an arrival rate $\lambda=\sum_{j=1}^{J} \lambda_{j}$, and a service rate $\mu_{\infty}$ equal to the arithmetic mean of each service rate: $\mu_{\infty}=\sum_{j=1}^{J} \alpha_{j} \mu_{j}$, where $\mu_{j}=\frac{C_{j}}{\mathbb{E}(\Sigma)}$ and $\alpha_{j}$ is the probability that a user is physically in zone $j$ (active or not) and is given by:

$$
\alpha_{j}=\prod_{i=1}^{j-1} \frac{\mathbb{E}\left(\Theta_{i+1}\right) P_{i i+1}}{\mathbb{E}\left(\Theta_{i}\right) P_{i+1 i}} \alpha_{1}, j=2, \ldots, J, \text { with } \sum_{i=1}^{J} \alpha_{i}=1 .
$$

Figure 3 illustrates this second bound in blue, corresponding to a PS queue with an equivalent capacity $C_{\infty}$ and users' average throughput $\gamma_{\infty}$ given by:

$$
C_{\infty}=\sum_{j=1}^{J} \alpha_{j} C_{j}, \text { and } \bar{\gamma}_{\infty}=C_{\infty}\left(1-\frac{\Lambda \mathbf{E}(\Sigma)}{C_{\infty}}\right) .
$$

The performance of the system where users have a given speed $V$, e.g., the throughput obtained by users during their sojourn in the cell, lies in between these two bounds. As illustrated in Figure 3, the throughput starts from a value $C_{V}$ when the load is very low, and converges to the limit $\frac{C_{\infty}}{\mathbb{E}(\Sigma)}$ (the same for any speed $V$ ) when the load is very high. In order to calculate the value of $C_{V}$, we consider the Continuous-Time Markov Chain (CTMC) represented in Figure 4 (for $J=4$ zones) and modeling the behavior of the system submitted to a very low load, i.e., when there is at most one customer in the cell. States $j$ of this CTMC corresponds to one customer in zone $j$, and state 0 to an empty system.

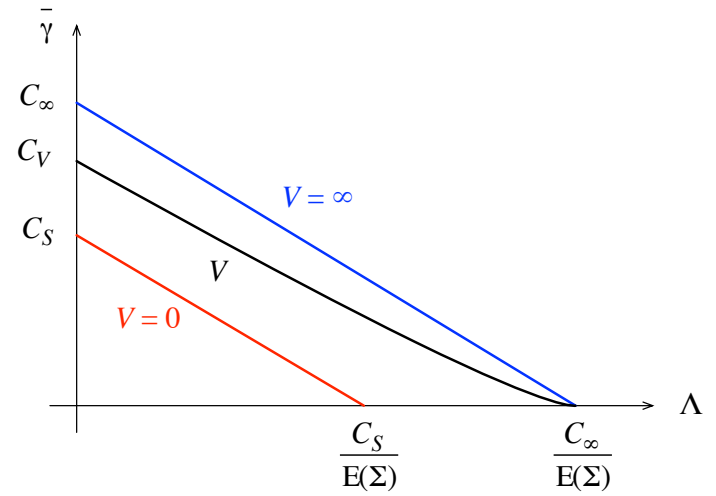

Fig. 3: Bounds on the throughput obtained by users in the cell

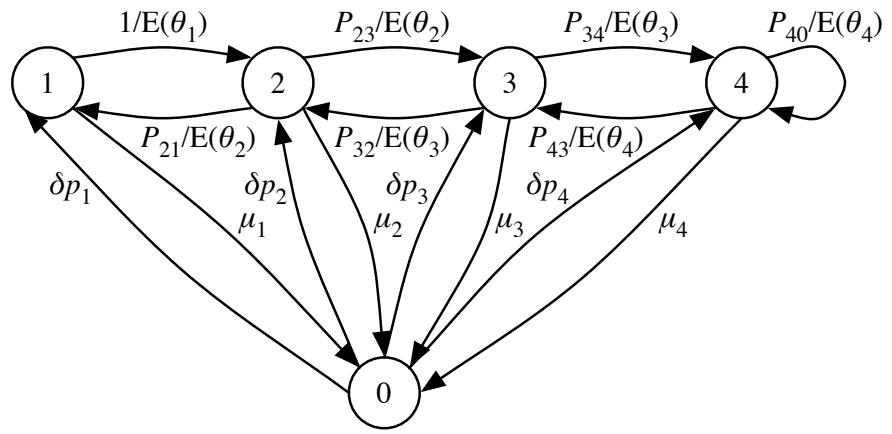

Fig. 4: CTMC modeling the cell at very low load

$C_{V}$ can be expressed from the stationary probability $\pi_{i}$ of this CTMC as in relation 24, and corresponds to the arithmetic mean of capacities $C_{j}$ weighted by the probabilities $\beta_{j}$ that an active user (supposed to be alone in the cell) is in zone $j$ :

$$
C_{V}=\sum_{j=1}^{J} \beta_{j} C_{j} \text { with } \beta_{j}=\frac{\pi_{j}}{\sum_{i=1}^{J} \pi_{i}}, j=1, \ldots, J .
$$

Note that this result does not depend on rate $\delta$ appearing in this CTMC. Finally, one can easily check that for any speed $V$ of users, $C_{S}<C_{V}<C_{\infty}, C_{V} \rightarrow C_{S}$ when $V \rightarrow 0$, and $C_{V} \rightarrow C_{\infty}$ when $V \rightarrow \infty$. Last but not least, we will see in Section IV that our model respects all of these bounds. 


\section{E. End to end performance of users}

We still consider the case of a network of statistically equivalent cells, and derive the end to end performance of mobile users, possibly making intra-cell and inter-cell handovers. Because the size $\Sigma$ of data to be transferred by users is supposed to be exponentially distributed, when a user makes a handover and leaves the cell without having completed its transfer, the data remaining to be transferred has the same distribution as the original one (memoryless property of the exponential distribution). As a result, the number $n_{h}$ of handovers a user has to make and the number $n_{c}$ of cells a user has to visit, before the completion of its transfer, are both geometrically distributed with parameter $H$ (starting from 0 for the first one and from 1 for the second one), with means given by:

$$
\bar{n}_{h}=\frac{H}{1-H} \text { and } \bar{n}_{c}=\frac{1}{1-H} .
$$

We denote by $t_{h}$ the duration of a handover procedure, i.e, the duration of the service interruption when a user change cell. We can estimate with relation 26 , the average end to end transfer time $\bar{T}$ of user, defined as the average time for a user to complete a full transfer, whatever the number of cells and the number of zones in each cell the user has visited during its transfer:

$$
\bar{T}=\bar{n}_{c} \bar{R}+\bar{n}_{h} t_{h}
$$

Finally, we obtain the average end to end throughput $\bar{\Gamma}$ a user obtains during its full transfer, as:

$$
\bar{\Gamma}=\frac{\mathbb{E}(\Sigma)}{\bar{T}} .
$$

\section{Performance Results}

We have compared the results provided by the models to those delivered by a home-made discrete-event simulator developed in Matlab. We reproduced in simulation the traffic assumptions and the mobility model described in Sections II-B. We assumed that the cell uses a number of 100 Ressource Blocks for the downlink channel and offers to users four MCS $(28,23,16,6)$. This results in four transmission zones with corresponding capacity $C_{1}=75 \mathrm{Mbit} / \mathrm{s}, C_{2}=51 \mathrm{Mbit} / \mathrm{s}$, $C_{3}=31 \mathrm{Mbit} / \mathrm{s}$ and $C_{4}=10 \mathrm{Mbit} / \mathrm{s}$ [15]. We set the constant $K=\frac{1}{\sqrt{\pi}}$ and we take $\mathbb{E}(\Sigma)=10 \mathrm{MB}$ for the mean data volume to be transferred by all users. We used the following radius corresponding to the concentric circles model of the cell: $R_{1}=100 \mathrm{~m}, R_{2}=150 \mathrm{~m}, R_{3}=200 \mathrm{~m}$ and $R_{4}=250$ $\mathrm{m}, R_{4}$ corresponds approximately to the operating range of LTE antenna in urban environment. The mean sojourn time in each zone $\mathbb{E}\left(\Theta_{j}\right)$ is given by equation 2 . According to the estimations given in Section II-C, resulting probability are reported in Table I.
TABLE I: Cells parameters

\begin{tabular}{|l|l|l|l|l|}
\hline parameters & zone 1 & zone 2 & zone 3 & zone 4 \\
\hline $\begin{array}{l}\text { new connection } \\
\text { probabilities } p_{j}\end{array}$ & 0.16 & 0.20 & 0.28 & 0.36 \\
\hline moving probabilities & $P_{12}=1$ & $P_{21}=0.33$ & $P_{32}=0.37$ & $P_{43}=0.40$ \\
$P_{i j}$ & & $P_{23}=0.67$ & $P_{34}=0.63$ & $P_{40}=0.60$ \\
\hline
\end{tabular}

\section{A. Validation}

This subsection Aims to validating the proposed model; to do this we consider one cell with $J=4$ zones (with above parameters).

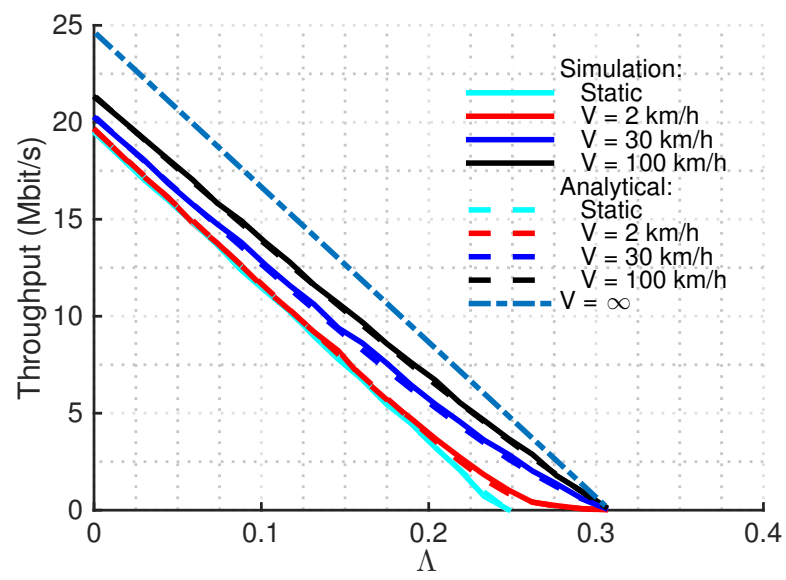

Fig. 5: Throughput obtained by active users as a function of the total arrival rate of new connection demands.

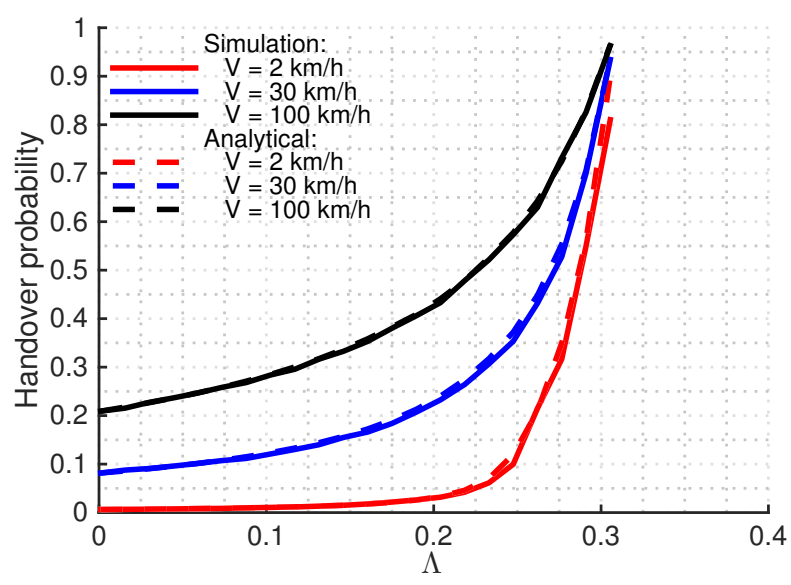

Fig. 6: Handover probability as a function of the total arrival rate of new connection demands.

Figure 5, 6 and 7 respectively show comparison of users' throughput, handover probability and users' sojourn time in the 


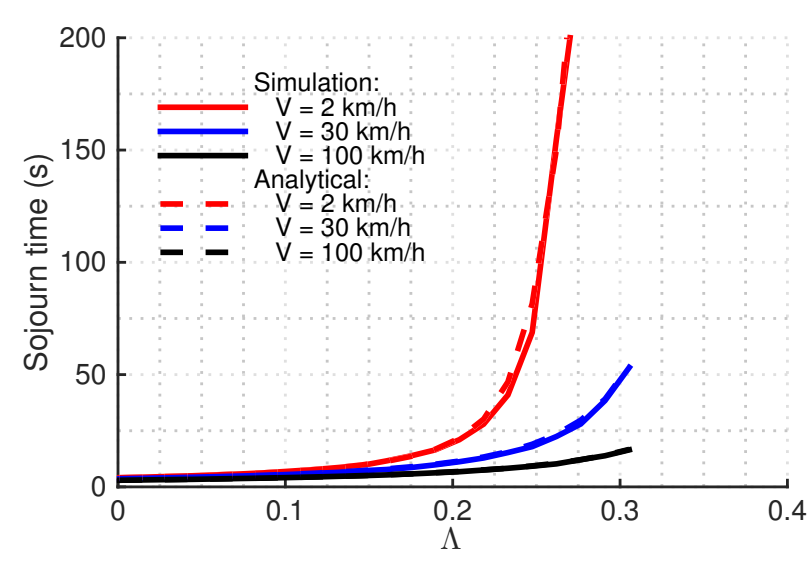

Fig. 7: Average sojourn time in considered cell as a function of the total arrival rate of new connection demands.

considered cell as a function of the total arrival rate of new connection demands $\Lambda$. We chose different values of speed $V$.

We observe that the predicted model is very close to simulation results. The average relative error between performances predicted by the model and those obtained by simulation is about $8 \%$ in average and this error remains less than $22 \%$ in the worst case.

As explained in section III-D, mobile users' throughput (Figure 5) is bounded by the throughput obtained when all users are static (lower bound) and the throughput obtained with infinite speed (upper bound). As expected, users mobility improve users' sojourn time (see Figure 7), capacity $C_{V}$, users' throughput and stability which is $\Lambda<0.24$ for the system with static users and $\Lambda<0.30$ when users are mobile. In this latter case, observe that the stability condition does not depend on users speed. The reason of this improvement is that, gain from users moving from a poor channel conditions to a good ones outweighs the loss from users moving in the reverse direction. Note that users in a favorable conditions have a better chance to complete their transfers before moving to a worse location.

It is important to observe that, of course, this improvement comes with an increase in the handover rate (see Figure 6). The handover $H$ starts from initial value that depends on speed and increases with $\Lambda$. $H$ converging to 1 when $\Lambda \rightarrow 0.30$.

\section{B. End to end performance}

We now investigate the end to end users' performance in the system.

Figure 8 show the number of visited cells in the network as a function of the total arrival rate of new connection demands $\Lambda$ for different values of users speed. As expected and in view of equation 25 and Figure 6; the number of visited cells is strongly impacted by the users' speed and is an increasing function of the arrival rate of new connection demands.

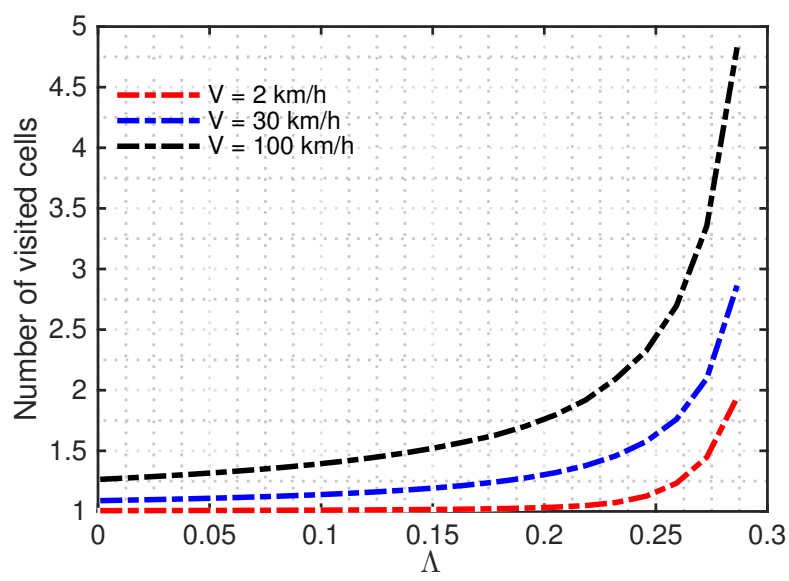

Fig. 8: Average number of visited cells as a function of the total arrival rate of new connection demands.

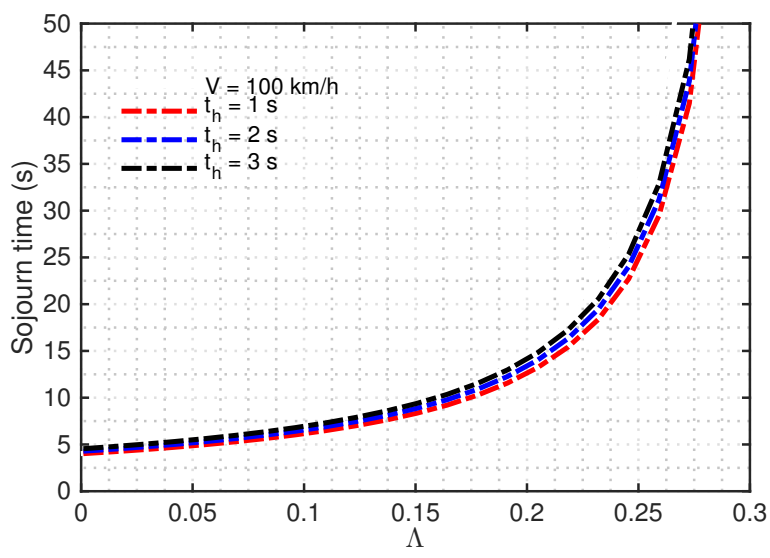

Fig. 9: Average sojourn time in the network as a function of the total arrival rate of new connection demands.

Since in LTE only hard handover is supported, the time required to complete a transfer must take account of service interruption due to handover procedure.

Figure 9 depicts users' sojourn time in the network (the time a user needs to complete a transfer) still as a function of the total arrival rate of new connection demands $\Lambda$. We chose three values for the duration of a handover procedure $t_{h}(1 \mathrm{~s}$, $2 \mathrm{~s}, 3 \mathrm{~s})$ and $100 \mathrm{~km} / \mathrm{h}$ for users speed.

Unsurprisingly, the time a user takes to complete a transfer increases with total arrival rate $\Lambda$. This time is also affected by the duration of the handover procedure.

\section{Impact of users mobility}

We now discuss the impact of users speed in performances. Figure 10 present, for a total arrival rate of new connexion demands $\Lambda=0.15$, the throughput as a function of users' speed. Note from this curve the significant gain of mobility compare to the scenario where all users are static. In particular, 
the throughput of mobile users is increasing function of the speed, bounded by static users throughput and the throughput obtain if users had infinite speed.

Figure 11 is the analog of Figure 10 with consideration of the duration of service interruption due to handover procedure which is set to $2 \mathrm{~s}$. This curve highlights the impact of handover on throughput gain. Specially, for speed less than $420 \mathrm{~km} / \mathrm{h}$, mobility leads to a throughput gain; and above this value, we observe a loss of throughput compared to scenario with only static users.

This numerical results show how handover rate counterbalance the flow-level performance improvement and prove that, mobility gain is a non-monotonic function of users' speed.

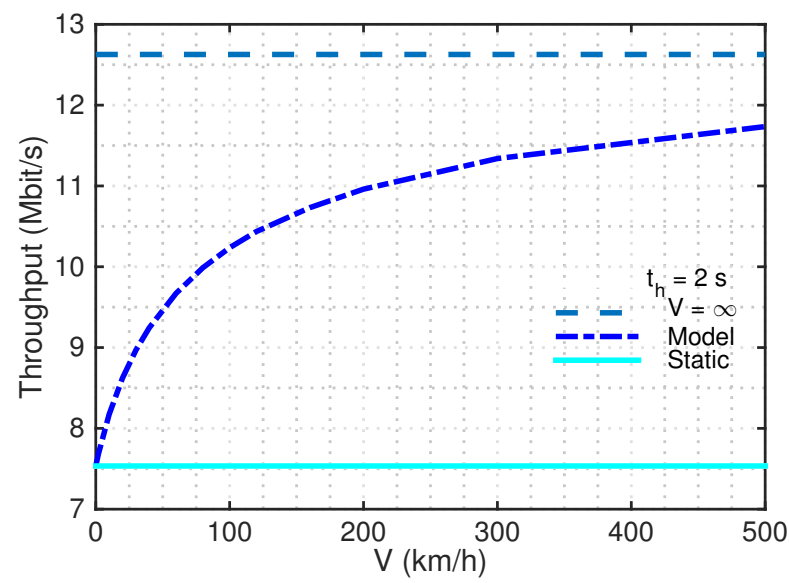

Fig. 10: Average Throughput obtain by active users as a function of users' speed when $\Lambda=0.15$.

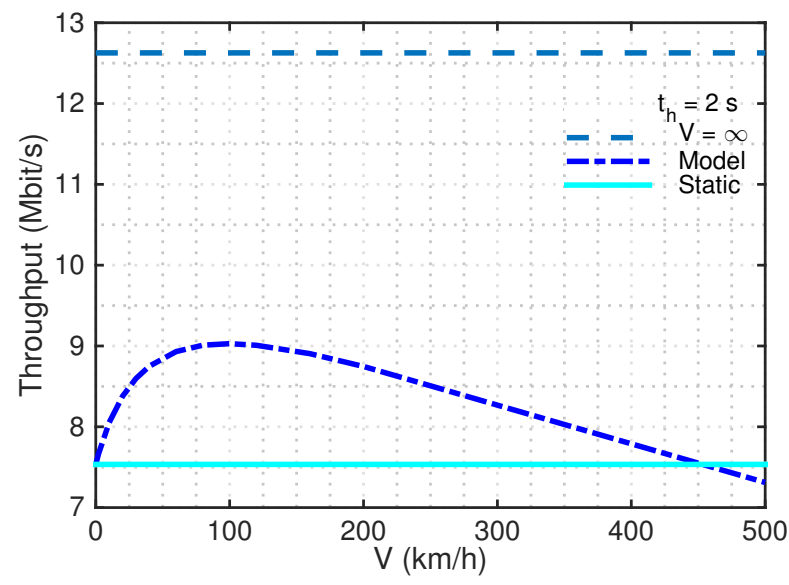

Fig. 11: Average Throughput obtain by active users as a function of users' speed taking into account the service interruption due to handover procedure when $\Lambda=0.15$.

\section{CONClusion}

We have developed a PS queue model for performance evaluation of data cellular networks with a round-robin policy, taking into account intra-and inter-cell mobility of users. We have shown that this model is consistent with known analytical bounds corresponding to static users or infinite speed, and provides a very good accuracy for more general speeds. Our model confirms that mobility may improve performance of users in a given cell, and enables to quantify the gain. It also provides end-to-end performance of users among a network of statistically equivalent cells, and shows that performance is not anymore a monotonic function of the speed. We believe that the approach used in this paper consisting in decoupling the mobility model from the cell model, can be applied to many other models or systems, e.g., coupled PS queues models for networks of interacting base stations, or models for more general scheduling policies.

\section{REFERENCES}

[1] A. J. Goldsmith and S.-G. Chua, "Adaptive coded modulation for fading channels," IEEE Transactions on Communications, 1998.

[2] U. Ayesta, M. Erausquin, and P. Jacko, "A modeling framework for optimizing the flow-level scheduling with time-varying channels," Performance Evaluation, vol. 67, no. 11, pp. 1014-1029, 2010.

[3] T. Bonald, "A score-based opportunistic scheduler for fading radio channels," in European Wireless, 2004.

[4] S. C. Borst, A. Proutiere, and N. Hegde, "Capacity of wireless data networks with intra-and inter-cell mobility.," in INFOCOM, 2006.

[5] T. Bonald, S. C. Borst, and A. Proutière, "How mobility impacts the flow-level performance of wireless data systems," in INFOCOM 2004. Twenty-third AnnualJoint Conference of the IEEE Computer and Communications Societies, IEEE, 2004.

[6] T. Bonald, S. Borst, N. Hegde, M. Jonckheere, and A. Proutiere, "Flowlevel performance and capacity of wireless networks with user mobility," Queueing Systems, 2009.

[7] S. C. Borst, N. Hegde, and A. Proutiere, "Mobility-driven scheduling in wireless networks," in INFOCOM 2009, pp. 1260-1268, IEEE, 2009.

[8] M. K. Karray, "User's mobility effect on the performance of wireless cellular networks serving elastic traffic," Wireless Networks, 2011.

[9] J. Kim, B. Kim, J. Kim, and Y. H. Bae, "Stability of flow-level scheduling with markovian time-varying channels," Performance Evaluation, vol. 70, no. 2, pp. 148-159, 2013.

[10] A. Jdidi and T. Chahed, "Flow-level performance of proportional fairness with hierarchical modulation in ofdma-based networks," Computer Networks, 2011.

[11] N. Abbas, T. Bonald, and B. Sayrac, "Opportunistic gains of mobility in cellular data networks," in 13th International Symposium on Modeling and Optimization in Mobile, Ad Hoc, and Wireless Networks (WiOpt), 2015, pp. 315-322, IEEE, 2015.

[12] T. Bonald and A. Proutière, "Wireless downlink data channels: user performance and cell dimensioning," in Proceedings of the 9th annual international conference on Mobile computing and networking, pp. 339352, ACM, 2003.

[13] C. Tarhini and T. Chahed, "Qos-oriented resource allocation for streaming flows in ieee802. 16e mobile wimax," Telecommunication Systems, vol. 51, no. 1, pp. 65-71, 2012.

[14] B. Baynat, R.-M. Indre, N. Nya, P. Olivier, and A. Simonian, "Impact of mobility in dense lte-a networks with small cells," in IEEE Vehicular Technology Conference (VTC Spring), pp. 1-5, IEEE, 2015.

[15] 3GPP, "3gpp ts 36.213 v9.2.0 (2010-06): Physical layer procedures," 2010. 